\title{
E-MAP-115, encoding a microtubule- associated protein, is a retinoic acid-inducible gene required for spermatogenesis
}

\author{
Masayuki Komada, ${ }^{1}$ Derek J. McLean, ${ }^{2}$ Michael D. Griswold, ${ }^{2}$ Lonnie D. Russell, $^{3}$ \\ and Philippe Soriano ${ }^{1,4}$ \\ ${ }^{1}$ Program in Developmental Biology, Division of Basic Sciences, Fred Hutchinson Cancer Research Center, Seattle, \\ Washington 98109 USA; ${ }^{2}$ Department of Biochemistry and Biophysics, Washington State University, Pullman, Washington \\ 99164 USA; ${ }^{3}$ Department of Physiology, Southern Illinois University School of Medicine, Carbondale, Illinois 62901 USA
}

Cell type-specific microtubules, such as the Sertoli cell microtubules and the manchette and flagellum microtubules of the spermatids, play essential roles in spermatogenesis. We identified the gene encoding E-MAP-115 (epithelial microtubule-associated protein of $115 \mathrm{kD}$ ) as a retinoic acid-inducible gene using gene trap mutagenesis in mouse embryonic stem cells. The gene trap insertion led to a null allele of the $E-M A P-115$ gene and, in agreement with its high expression in the testis, male mice homozygous for the mutation were sterile because of deformation of spermatid nuclei and subsequent gradual loss of germ cells. Consistent with a possible role for E-MAP-115 in stabilizing microtubules, microtubule associations in the mutant were morphologically abnormal in the manchette of spermatids and in Sertoli cells. We hypothesize that the abnormal microtubules in these two cell types are responsible for deformation of spermatid nuclei and germ cell loss, respectively, and indicate an essential role for E-MAP-115 in microtubule functions required for spermatogenesis.

[Key Words: E-MAP-115; gene trap; microtubule; retinoic acid; spermatogenesis]

Received February 22, 2000; revised version accepted April 6, 2000.

Vitamin A (retinol) deficiency causes a number of defects in adult animals as well as in embryos (Wolbach and Howe 1925; Wilson et al. 1953). All of these defects, except for blindness caused by photoreceptor degeneration, can be reversed by administration of retinoic acid (RA), a metabolite of vitamin A, indicating that RA is the biologically active derivative of vitamin A (Thompson et al. 1964; van Pelt and de Rooij 1991). RA binds and activates two types of receptor families, retinoic acid receptor (RAR) and retinoid $X$ receptor (RXR), both of which consist of three members: $\alpha, \beta$, and $\gamma$ (for review, see Chambon 1996). Almost all the vitamin A deficient (VAD) phenotypes are recapitulated in knockout mice with mutations in $R A R$ and $R X R$ genes, indicating that the biological effects of RA are exerted through these receptors (for review, see Kastner et al. 1995).

Although the physiological functions of RARs and RXRs have been studied extensively, little is known about their target genes that are required for exerting RA actions in vivo. To identify RA target genes and examine their physiological roles, we utilized gene traps in mouse embryonic stem (ES) cells as a tool to identify RA-responsive genes and mutate them at the same time. In

${ }^{4}$ Corresponding author.

E-MAIL psoriano@fhcrc.org; FAX (206) 667-6522. gene trap mutagenesis, expression of the introduced reporter gene only occurs when it is inserted downstream of a cellular promoter because it lacks its own promoter (Gossler et al. 1989; Friedrich and Soriano 1991; von Melchner et al. 1992). As reporter gene expression is regulated by the trapped promoter, comparison of its expression level in the presence and absence of RA can be used to screen for gene trap events in RA-responsive genes (Forrester et al. 1996). Performing this screen in ES cells permits transmission of the gene trap insertion through the germ line and analysis of the loss-of-function phenotype of the trapped gene in mice homozygous for the insertion (Friedrich and Soriano 1991; Skarnes et al. 1992; von Melchner et al. 1992). We characterized a gene trap mutation in an RA-inducible gene, E-MAP-115 (epithelial microtubule-associated protein of $115 \mathrm{kD}$ ).

Microtubules (MTs) are a major component of the cytoskeleton essential for a number of cellular functions, including positioning and transport of organelles and vesicles, maintenance of cell shape and polarity, cell motility, and cell division. They are a dynamic structure of which assembly/disassembly is controlled by polymerization/depolymerization of $\alpha$ - and $\beta$-tubulin subunits. Different types of MTs contain different MT-associated proteins (MAPs) and these MAPs regulate the dynamics or stability of MTs (for review, see Hirokawa 1994; Man- 
delkow and Mandelkow 1995|. E-MAP-115 was identified as a MAP in HeLa cells (Masson and Kreis 1993). It is a unique protein with no homology to other proteins in the databases. It is localized to a perinuclear subdomain of MTs, and is abundant in cultured cell lines of epithelial origin but is not expressed in fibroblasts (Masson and Kreis 1993). Faire et al. (1999) recently reported that E-MAP-115 is localized uniformly along the length of all MTs. In mice, E-MAP-115 transcripts are highly expressed in the testis and kidney, and at lower levels in many other tissues (Fabre-Jonca et al. 1998). Several lines of evidence implicate E-MAP-115 in stabilizing and reorganizing MTs. First, overexpression of E-MAP-115 stabilizes MTs against nocodazole, an MT-depolymerizing agent (Masson and Kreis 1993). Second, expression of EMAP-115 increases in polarized epithelial cells that have undergone MT reorganization, such as terminally differentiating keratinocytes (Fabre-Jonca et al. 1999). Finally, association of E-MAP-115 with MTs decreases at the onset of mitosis when MTs become dynamic (Masson and Kreis 1995). This decrease is correlated with serine and threonine phosphorylation of the protein, suggesting that phosphorylation of E-MAP-115 regulates its MTbinding affinity (Masson and Kreis 1995).

We show that E-MAP-115 null mice exhibit two defects in spermatogenesis: spermatid deformation in the first wave of spermatogenesis and subsequent germ cell loss. These defects were associated with morphologically abnormal MTs in the manchette of the spermatids and in the Sertoli cells, indicating an essential role for E-MAP-115 in the function of MTs in these cells.

\section{Results}

\section{Screening for RA-responsive gene traps in ES cells}

The retroviral gene trap vector used in this study, ROSA $\beta g e o^{\star}$, is similar to the previously described vector ROSA $\beta g e o$, which has a $\beta g a l / n e o$ fusion gene $(\beta g e o)$ as a reporter (Friedrich and Soriano 1991). In ROSAßgeo ${ }^{\star}$, a mutation in the neo moiety (Yenofsky et al. 1990) has been corrected, resulting in higher neomycin phosphotransferase activity. ES cells were infected with ROSAßgeo* and selected with G418. As expression of $\beta g e o^{\star}$ relies on the activity of the trapped promoter, all G418-resistant clones represent gene trap events and should be $\beta$-gal-positive. With the original ROSA $\beta$ geo vector, most G418-resistant colonies indeed exhibited X-gal staining (Friedrich and Soriano 1991). With

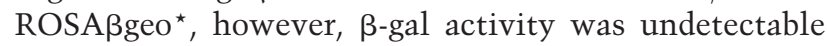
by X-gal staining in about $40 \%$ of colonies, reflecting the higher sensitivity of neo than $\beta$-gal activity. This allowed us to screen for cells in which the $\beta g e 0^{*}$ expression level is changed by RA treatment. Individual G418resistant colonies were picked, trypsinized, and plated in duplicate (see Materials and Methods for details). Cells in one plate were treated with $1 \mu \mathrm{M}$ all-trans RA for $24 \mathrm{hr}$ and compared to the untreated control by staining with $\mathrm{X}$-gal. Clones in which the expression of the trapped gene is up-regulated by RA will be white or light blue (undetectable or low $\beta$-gal activity) in the absence of RA and turn dark blue (high $\beta$-gal activity) following RA stimulation (Fig. 1). Conversely, clones in which the expression of the trapped gene is down-regulated by RA will be dark blue in the absence of RA and turn light blue after stimulation.

Of 612 screened colonies, $\beta$-gal activity was up-regulated in 49 clones and down-regulated in five clones after RA treatment. The induction/repression was confirmed by Northern blot analysis in some of these clones including the one characterized in this study. As part of an ongoing gene trap screen for developmental mutants in our laboratory, this clone was designated ROSA63. In Fig. 2A, ROSA63 ES cells were stimulated with RA for various time periods and RNAs were probed with $\beta$-gal. Expression of $\beta g e O^{*}$ was up-regulated $\sim$ fivefold after $6 \mathrm{hr}$ of RA stimulation and the induced level persisted for at least $24 \mathrm{hr}$. These results confirmed that the expression of the trapped gene is transcriptionally regulated by RA. We focused on ROSA63 in this study because the mutant mice exhibited a phenotype similar to those in VAD animals and $R A R \alpha$ knockout mice (see below).

\section{Derivation and male sterility of ROSA63 mutant mice}

ROSA63 germ line chimeric mice were derived and crossed to $129 / \mathrm{Sv}$ and C57BL/6J mice to derive mutant mice. Heterozygous offspring did not display an overt

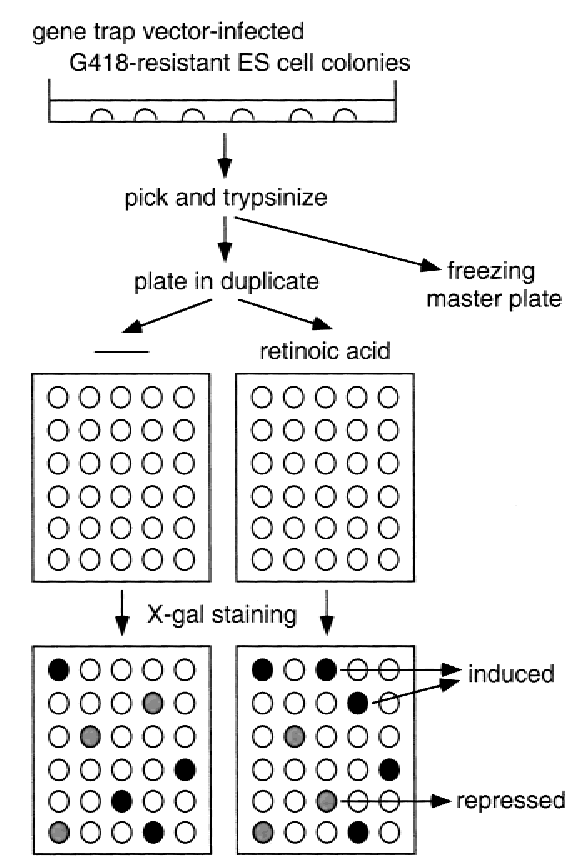

Figure 1. Schematic diagram of screening for RA-responsive gene traps. ES cells were infected with a retroviral vector

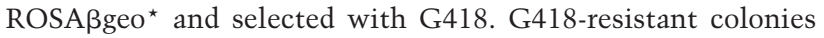
were picked, trypsinized, plated in duplicate, and stimulated with or without RA. After stimulation, the cells were stained with X-gal, and $\beta$-gal activity was compared between unstimulated and stimulated cells. 
Figure 2. Induction of E-MAP-115 expression by $\mathrm{RA}$ and no $E$-MAP-115 expression in ROSA63 mutant testis. (A) Time course of induction of $\beta g e o^{*}$ expression by RA in ROSA63 ES cells. Cells were serum-starved, and stimulated with RA for indicated time periods. Transcripts were detected by Northern blot analysis with a $\beta$-gal probe. Ethidium bromide staining of $28 \mathrm{~S}$ ribosomal RNA is shown below as a loading control. (B) Southern blot analysis of DNAs from tail biopsies of wild-type $(+/+)$, heterozygous $(+\mid-)$, and homozygous $(-/-)$ mutant mice with mixed probes of neo and wnt1. The intensity of the neo-positive band, absent in the wild type, is twice as high in the homozygote as in the heterozygote. The wnt1 genomic probe was used as an internal standard (i.s.). (C) Northern blot analysis of RNAs from wild-type $(+/+)$, heterozygous $(+/-)$, and

A
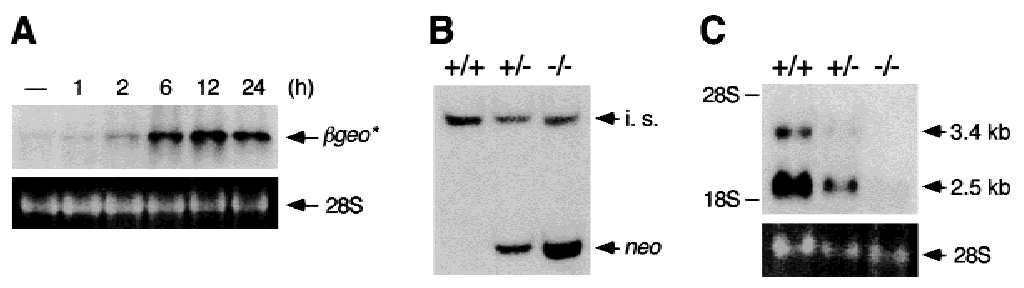

D

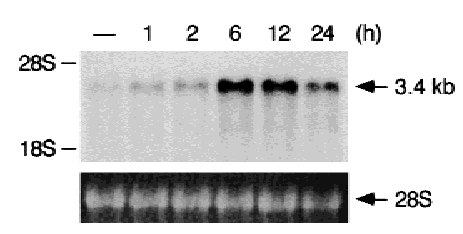

E

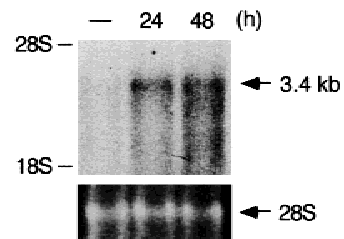

homozygous (-/-) mutant testes with E-MAP-115 cDNA. The broadly expressed 3.4-kb and the testis-specific 2.5 -kb transcripts were not detected in the homozygote. The positions of the $18 \mathrm{~S}$ and $28 \mathrm{~S}$ ribosomal RNAs are indicated on the left. $(D)$ Time course of induction of endogenous E-MAP-115 expression by RA in wild-type ES cells. The experiment was performed as described in $A$ with $E-M A P-115$ cDNA as a probe. (E) Induction of E-MAP-115 expression by retinol in the small intestine of VAD mice. RNAs from small intestines of VAD mice administered with or without retinol for $24 \mathrm{hr}$ or $48 \mathrm{hr}$ were probed with E-MAP-115 cDNA.

phenotype and were fertile. Mice were then collected from the intercrossing of heterozygous parents and genotyped by Southern blot analysis by monitoring the intensity of the gene trap-specific neo-positive band (Fig. 2B). Mice of all genotypes were recovered according to Mendelian expectations, and homozygotes appeared grossly normal. However, test matings with wild-type and heterozygous mutant mice demonstrated that whereas the homozygous females had normal fertility, the homozygous males were sterile over a 3-month breeding period despite their ability to copulate as judged by the presence of vaginal plugs. The same phenotype was observed both on congenic $129 / \mathrm{Sv}$ and mixed $129 / \mathrm{Sv} \times \mathrm{C} 57 \mathrm{BL} / 6 \mathrm{~J}$ genetic backgrounds.

\section{Mutation in the E-MAP-115 gene in ROSA63 mutant} mice

A ROSA63/Bgeo* fusion cDNA was cloned from ROSA63 ES cells by $5^{\prime}$ RACE, and a 188-bp sequence upstream of $\beta g e o^{*}$ was determined. The 67-bp sequence just upstream of $\beta g e O^{*}$ was identical to the sequence of the coding region (methionine residue 1 to alanine residue 23) of mouse E-MAP-115 cDNA (Fabre-Jonca et al. 1998). Although the $5^{\prime}$ noncoding region of mouse $E$ MAP-115 cDNA has not been reported, the sequence upstream of the 67-bp sequence was similar to that of the $5^{\prime}$ noncoding region of human E-MAP-115 cDNA, indicating that the gene trap vector integrated into the $E$ MAP-115 gene. The vector was inserted into a site that encodes the alanine residue 23 , suggesting that the amino-terminal 23-amino acid peptide is produced as an in-frame fusion protein with $\beta g e{ }^{\star}$. To determine whether a transcript that encodes a region downstream of the gene trap insertion site is expressed in the mutant, RNAs from wild-type, heterozygous, and homozygous mutant testes were probed by Northern blot analysis us- ing an E-MAP-115 cDNA fragment corresponding to the amino acid residues 229-571. A broadly expressed 3.4-kb transcript as well as a testis-specific $2.5-\mathrm{kb}$ transcript, which is not expressed in other tissues and ES cells (Figs. 2D,E), was detected in the wild-type and the heterozygous mutant (Fig. 2C; Fabre-Jonca et al. 1998). In the homozygous mutant, no E-MAP-115 transcripts were detected (Fig. 2C), although by longer exposure, very low expression of the testis-specific transcript was detectable (not shown). It is unlikely that this transcript is translated to a protein because no smaller E-MAP-115 isoform can be detected by immunoblot analysis in the testis (Fabre-Jonca et al. 1998). As the MT-binding domain, the only known functional domain of the protein, does not map to the amino-terminal 23-amino acid region (Masson and Kreis 1993), this gene trap insertion most likely led to a null allele of the gene.

\section{Induction of E-MAP-115 expression by retinoid in cultured cells and in vivo}

Induction of E-MAP-115 by RA was examined by Northern blot analysis in wild-type ES cells. It exhibited a similar profile as the $\beta g e o^{*}$ induction in ROSA63 ES cells, although the mRNA level decreased to about $65 \%$ of the maximal level after $24 \mathrm{hr}$ stimulation (Fig. 2D).

We next examined whether the expression is induced by RA in vivo. We used RA-starved VAD mice, as cells in normal mice are exposed to endogenous RA. VAD mice were administered with or without retinol (vitamin A), and E-MAP-115 expression was examined in several tissues. Among the tissues tested, the expression was upregulated fivefold in the small intestine by $24 \mathrm{hr}$ after retinol administration (Fig. 2E). Up-regulation was not observed in other tissues such as kidney (not shown), suggesting that E-MAP-115 induction by RA is specific to certain tissues or cell types. 
Jackson BSS Chromosome 10

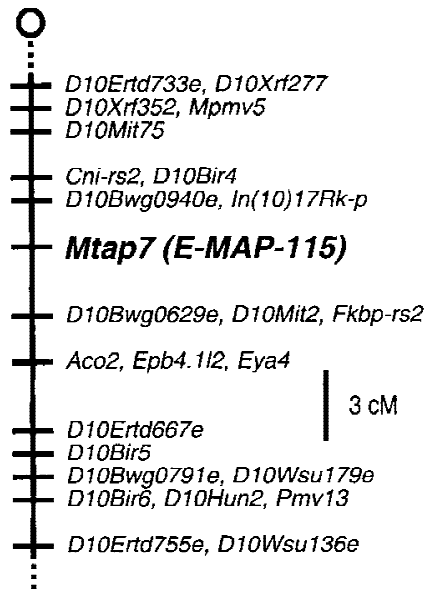

B Jackson BSS Chromosome 10
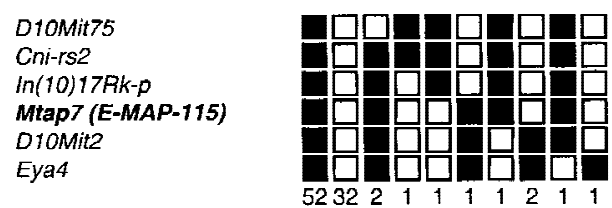

R SE $2.13 \quad 1.49$ $1.06 \quad 1.06$ $2.13 \quad 1.49$ $3.19 \quad 1.81$ $2.13 \quad 1.49$

Figure 3. Chromosomal localization of mouse E-MAP-115 (locus symbol Mtap7). (A) Map figure showing the proximal end of chromosome 10 with loci linked to E-MAP-115. The map is depicted with the centromere toward the top. A 3-cm scale bar is shown to the right. Loci mapping to the same position are listed in alphabetical order. $(B)$ Haplotype figure showing the proximal end of chromosome 10. Loci are listed in order with the most proximal at the top. (ロ) C57BL/6JEi allele; ( $\square$ ) SPRET/ Ei allele. The number of animals with each haplotype is given at the bottom of each column of boxes. The percent recombination (R) between adjacent loci is given to the right, with the standard error (S.E.) for each R. Missing typings were inferred from surrounding data when assignment was unambiguous. Raw data from the Jackson Laboratory were obtained from the World Wide Web address http://www.jax.org/resources/documents/ cmdata.

\section{Chromosomal localization of the E-MAP-115 gene}

About $30 \%$ of human male infertility has a genetic origin (McLachlan et al. 1998). Three infertility genes, two of which encode RNA-binding proteins, have been mapped to the human Y chromosome (for review, see Elliott and Cooke 1997). However, no autosomal gene has been identified as an infertility gene in human. To help understand the genetics of infertility, we determined the chromosomal localization of the mouse $E$ $M A P-115$ gene by an interspecific backcross analysis (see Materials and Methods for detail). The gene was mapped to the proximal region of mouse chromosome 10, which is syntenic with human chromosome 6q22-25 (Fig. 3; locus symbol Mtap7 in Mouse Genome Database).

\section{Expression of E-MAP-115 in mouse embryos and testis}

As $\beta$ geo $^{\star}$ expression in the mutant relies on the activity of the E-MAP-115 promoter, its expression pattern was examined by X-gal staining in heterozygous mutant embryos and also in the adult testis where the homozygous mutant exhibits a phenotype (see below).

During development, $\beta$-gal activity was detected in many epithelial cell types. At embryonic day 10.5 (E10.5), $\beta$-gal expression was restricted to the lens vesicle of the eye, otic vesicle, surface ectoderm of the nasal placode and branchial arches, apical ectodermal ridge (AER), gut epithelia, and the floor plate of the neural tube (Fig. 4A). At E12.5 and E14.5, E-MAP-115-expressing epi-
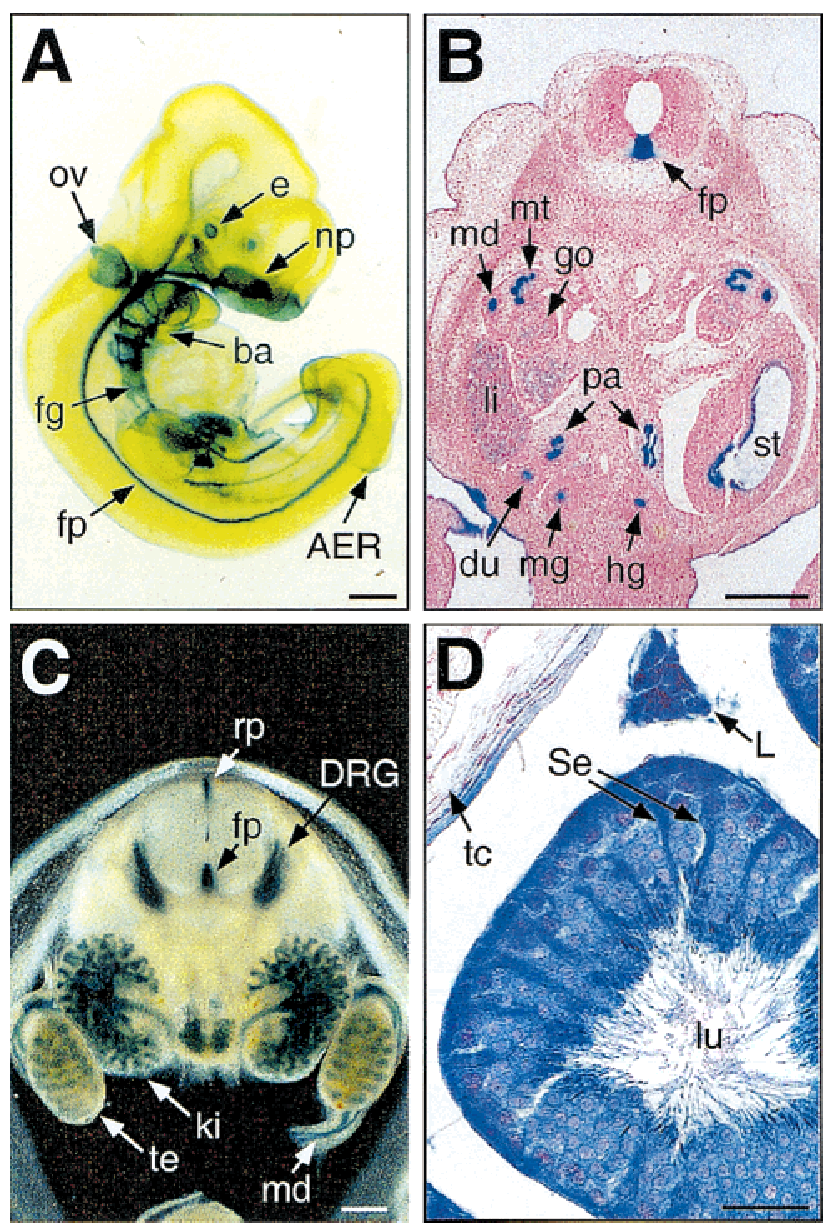

Figure 4. E-MAP-115 expression in mouse embryos and testis. E-MAP-115 expression was examined in ROSA63 heterozygous mutants by X-gal staining. (A) E10.5 embryo. (ba) Branchial arch; (e) eye; (fg) foregut; (fp) floor plate; (np) nasal placode; (ov) otic vesicle. Transverse sections of E12.5 (B) and E14.5 (C) embryos at the kidney/gonad level. The E12.5 embryo section was counterstained with Nuclear Fast Red. The E14.5 embryo was cut using a vibratome prior to staining. (du) Duodenum; (go) gonad; (hg) hindgut; (ki) kidney; (li) liver; (md) mesonephric duct; (mg) midgut; (mt) mesonephric tubule; (pa) pancreas; (rf) roof plate; (st) stomach; (te) testis. (D) A 3-month-old testis. (L) Leydig cell; (Se) Sertoli cell; (lu) lumen of the seminiferous tubule; (tc) testis capsule. Artificial shrinkage of the seminiferous tubule occurred due to mild fixation to protect the $\beta$-gal activity. Although some background staining was detected in Leydig cells from the wild-type testis, the staining was much darker in the heterozygote. Bars, $300 \mu \mathrm{m}(A-C) ; 30 \mu \mathrm{m}(D)$. 
thelia included those of the kidney, including the mesonephric duct and tubule, gut, stomach, ducts of the pancreas, bronchi of the lung, trachea, esophagus, ducts of the submandibular gland, whisker follicles, and surface ectoderm of the tongue and trunk (Fig. 4B,C; not shown). At E14.5, very dark staining was also observed in the dorsal root ganglia (DRG) and trigeminal ganglia (Fig. 4C; not shown). Whereas staining in the gonad was weak in E12.5 male (Fig. 4B) as well as female (not shown) embryos, staining in the testicular cord, a primitive seminiferous tubule consisting of Sertoli cells and germ cells, was apparent in the testis at E14.5 (Fig. 4C). The predominant X-gal staining in epithelial cell types but not in the surrounding mesenchyme was consistent with the previous observations obtained by in situ hybridization (Fabre-Jonca et al. 1998).

In the adult testis, E-MAP-115 is reported to be expressed broadly in germ cells, Sertoli cells, and Leydig cells (Fabre-Jonca et al. 1998). In agreement with this, $\beta$-gal activity was detected in all these cell types in the heterozygous mutant (Fig. 4D). The cytoplasmic processes of Sertoli cells generally exhibited darker staining (Fig. 4D), suggesting that this is the site of highest $E$ MAP-115 expression in the seminiferous tubule.

\section{Smaller testis in ROSA63 mutant mice}

Tissues which express relatively high levels of E-MAP115, such as kidney, lung, and small intestine, were of normal size and did not exhibit any histological abnormalities in ROSA63 homozygous mutant mice (not shown). In contrast, when dissected at 3 months of age, the homozygous mutant testes were less than one-third of the size of those of the heterozygous mutant littermates, with an average weight of $31 \pm 1 \mathrm{mg}(n=6)$, compared to $109 \pm 6 \mathrm{mg}(n=8)$ for the heterozygotes (Fig. $5 \mathrm{~A}, \mathrm{~B})$, although there was no significant difference in the body weights. The smaller size of the testis was already detectable by five weeks of age (not shown). The epididymis was also smaller in the homozygous mutant, but vas deferens, prostate, and seminal vesicle were of normal size and morphology (Fig. 5A; not shown).

\section{Spermatid deformation in the first wave}

of spermatogenesis in ROSA63 mutant testis

The histology of the mutant testis was examined in sections by light and electron microscopy. In mice, the first wave of spermatogenesis completes at five weeks of age. No overt abnormality was detected in the homozygous mutant testis at two or three weeks of age prior to the appearance of spermatids in the seminiferous tubule (not shown; Fig. 7F,G).

The first indication of testicular effects was seen during the first wave of spermatogenesis when round spermatids began their elongation phase of development. Light microscopy of 5-week-old mutant testis sections showed that, whereas round spermatids appeared normal (Fig. 5D), elongation, which takes place subsequent to
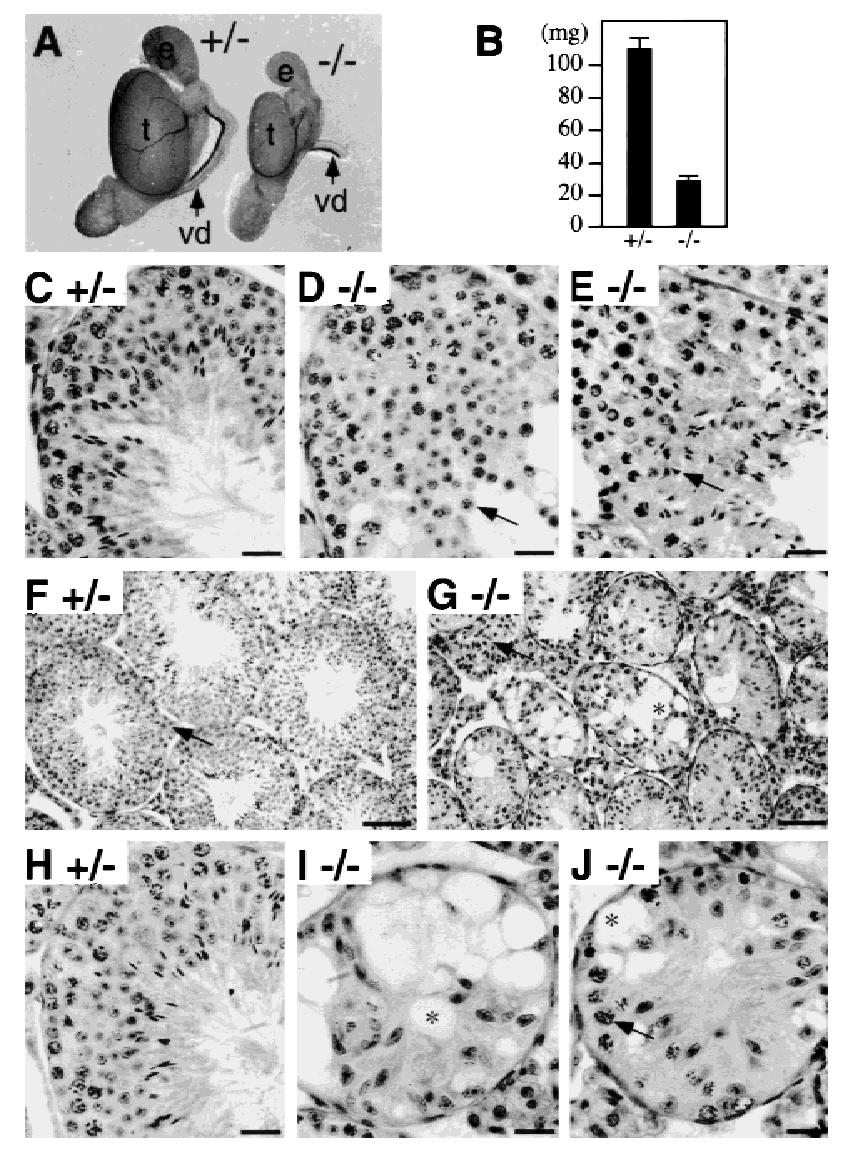

Figure 5. Testis phenotype of ROSA63 mutant mice. (A) Morphology of testes from 3-month-old heterozygous control $(+/-)$ and homozygous (-/-) mice. (e) Epididymis; (t) testis; (vd) vas deferens. (B) Weight of testes from 3-month-old heterozygous control (+/ $)$ and homozygous (-/ -$)$ mice. Mean \pm S.D. are shown ( $n=8$ for heterozygotes, $n=6$ for homozygotes). $(C-E)$ PAS and hematoxylin staining of 5 -week-old heterozygous control $(C)$ and homozygous $(D, E)$ testis sections at high magnification. Arrows in $D$ and $E$ indicate round and elongating spermatids, respectively. (F-J) PAS and hematoxylin staining of 3-month-old heterozygous control $(F, H)$ and homozygous $(G, I, J)$ testis sections at low $(F, G)$ and high $(H-J)$ magnification. Arrows in $F$ and $G$ indicate Leydig cells. An arrow in $J$ indicates a pachytene spermatocyte. Sertoli cell-associated vacuoles are also indicated $\left(^{*}\right)$ in $G, I$, and $J$. Bars, $25 \mu \mathrm{m}(C-E, H-J) ; 100 \mu \mathrm{m}(F, G)$.

step eight of spermiogenesis, was abnormal (Fig. 5E). Elongating spermatids in the first wave exhibited deformation of nuclei and underwent progressive degeneration at Stages XII, I, II, and III. Electron microscopy also showed that the shape of spermatids, particularly that of nuclei, was slightly abnormal at steps eight and nine of spermiogenesis and became more abnormal in mid-step 9-11 (Fig. 6). All these abnormalities were related to the presence and position of the manchette, a "skirt" of MTs enclosing the nonacrosomal portion of spermatids (Russell et al. 1991; Fig. 6A). MTs of the manchette are interlinked and linked to the nuclear envelope by an unknown mechanism (Russell et al. 1991). About $80 \%$ of sections showed one or more of the following abnormali- 


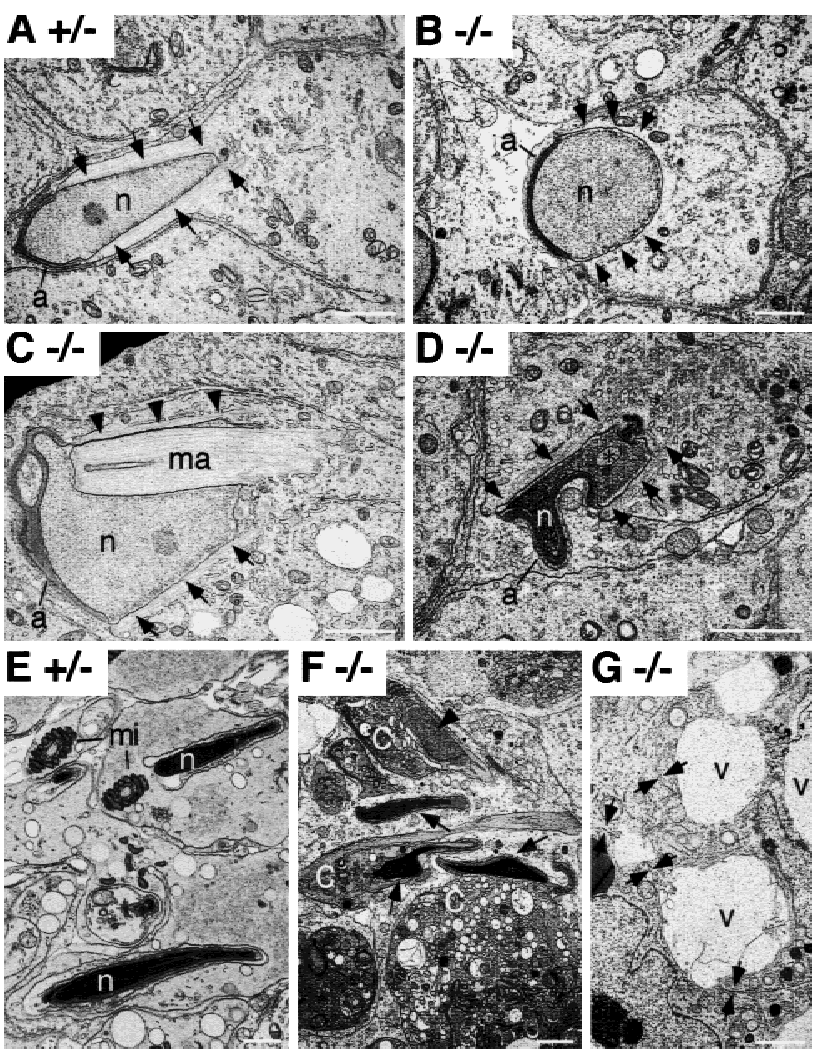

Figure 6. Electron microscopy of ROSA63 mutant testis. (A) A step 9 heterozygous control $(+/-)$ spermatid showing the expected configuration of the nucleus (n) and associated manchette (arrows). Note that the nuclear contour in the region of the manchette is always parallel with the manchette MTs. The normal manchette extends from the margins of the acrosome (a) into the caudal cytoplasm. $(B)$ The manchette is absent in this late step 8 homozygous mutant $(-/-)$ spermatid. As a result, the nuclear contour is generally rounded. The expected sites of the manchette are indicated by arrows. $(C)$ In this step 10 homozygous mutant $(-/-)$ spermatid, the manchette is positioned partially normally (arrows) but also indented the nucleus (ma). On one aspect of the spermatid, it is greatly reduced (arrowheads). Consequently, the shape of the spermatid head is grossly abnormal. (D) A step 13 homozygous mutant (-/-) spermatid showing an abnormally shaped condensing nucleus. Although the manchette skirts the nucleus (arrows), there is an indentation of the nucleus by a bundle of manchette MTs $\left(^{\star}\right)$. (E) Step 16 heterozygous control spermatids. Mitochondria in the midpiece of spermatids (mi) are indicated. (F) Degenerating step 15 homozygous mutant (-/-) spermatids. Abnormal (arrows) and developmentally arrested (arrowhead) nuclei are noted. In all spermatids, the cytoplasm (c) condensed during the degenerative process. $(G)$ Ultrastructural appearance of Sertoli cells in the homozygous mutant (-/-). Extracellular vacuoles with a proteinaceous content $(\mathrm{v})$ are seen between Sertoli cells. Evidence that the vacuoles are between Sertoli cells is the visualization of the Sertoli cell plasma membranes opening to form the vacuoles (arrows). Bars, $2 \mu \mathrm{m}$.

ties: absence or greatly reduced manchette MTs along the nuclear envelope (Fig. 6B); ectopic manchette along regions of the nucleus that normally do not display manchette (Fig. 6C); and ectopic manchette along the plasma membrane (not shown). Regions of the nucleus without manchette were generally rounded (Fig. 6B) and regions of the nucleus impacted by ectopic manchette were indented in abnormal ways (Fig. 6C). Close examination of individual manchette MTs in the mutant, however, showed no apparent difference in appearance or interconnections from those in control mice (not shown). As spermiogenesis proceeded, the abnormal shapes taken by elongating nuclei were also found in condensing spermatids (Fig. 6D). Compared to control spermatids (Fig. 6E), all condensed spermatids demonstrated abnormal shapes and were phagocytozed in early stages of the cycle by adjoining Sertoli cells in the mutant (Fig. 6F). The flagellum, the other MT-rich structure in spermatids, ap-
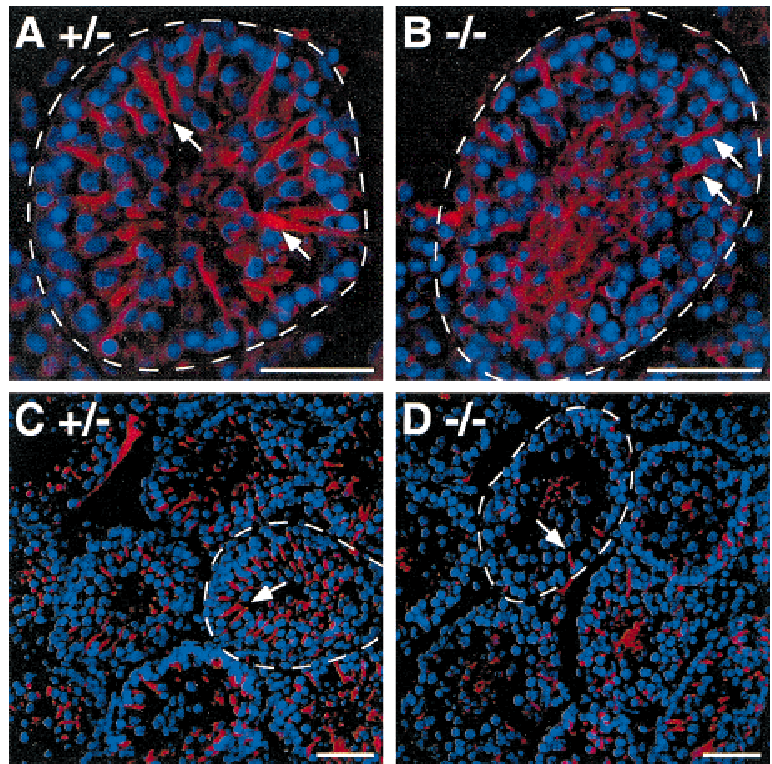

E

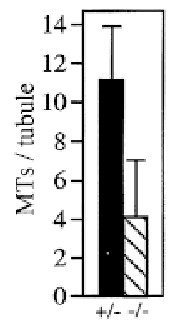

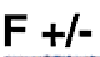

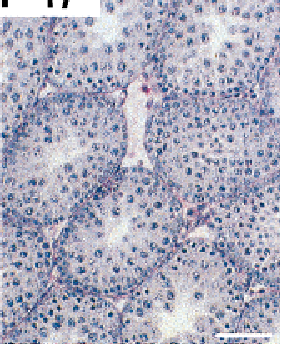

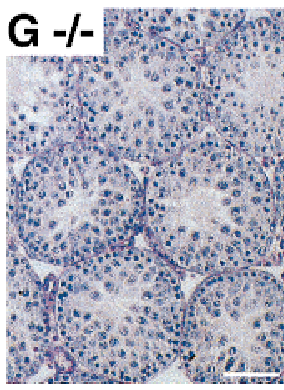

Figure 7. Morphology of the Sertoli cell MTs in ROSA63 mutant testis. $(A-D)$ Testis sections from 3-week-old heterozygous control $(A, C)$ and homozygous $(B, D)$ mice were stained with anti- $\beta$-tubulin antibody $($ red $) .(A, B)$ High magnification; $(C, D)$ low magnification. Nuclei of cells were costained with DAPI (blue). Basement membranes of the seminiferous tubules are indicated by broken lines. Arrows indicate Sertoli cell MT bundles. Note that they are not as well developed and fewer in the mutant. (E) Numbers of detectable Sertoli cell MT bundles in heterozygous control $(+/-)$ and homozygous $(-/-)$ testes. Mean numbers per seminiferous tubule $(n=20$ for each genotype) are shown with S.D. $(F, G)$ PAS and hematoxylin staining the other testes of the heterozygous control $(F)$ and homozygous $(G)$ mice used for the experiments in $A-E$. Note that the homozygous testis is histologically normal at this age. Bars, $50 \mu \mathrm{m}$. 
peared normal by electron microscopy in the mutant (not shown), in contrast to the machette.

\section{Gradual germ cell loss in ROSA63 mutant testis}

The second indication of testis effects was already evident at five weeks of age in many seminiferous tubule sections (not shown), and the phenotype became more severe as the mice grew older. At three months of age, the diameter of the seminiferous tubule was significantly shorter in the homozygous testis, and the lumen of the tubules were filled with a mass of Sertoli cells (Fig. $5 F, G)$. Extracellular vacuoles associated with the Sertoli cells were observed frequently in the homozygous mutant tubules (Fig. 5G). These vacuoles were found between Sertoli cells and were bounded by Sertoli-Sertoli tight junctional complexes (Fig. 6G). In addition, Leydig cell hyperproliferation was apparent (Fig. 5F,G). This is likely a secondary defect owing to the shrinkage of seminiferous tubules. In some of the homozygous mutant tubules, germ cells were completely absent (Fig. 5I). In other tubules, some spermatogonia and spermatocytes (up to pachytene stage) were noticed (Fig. 5J). Round spermatids were observed in very few tubules (not shown) and elongated spermatids were never present. Spermatogenesis was mostly blocked by midmeiosis at this age. By 12 months of age, most of the cells in homozygous mutant tubule sections were Sertoli cells, although occasional spermatogonia were still present in some tubules (not shown). This gradual loss of germ cells indicated that the loss is not germ-cell stage specific. Such a pattern of loss suggests that it is due to a problem of supporting Sertoli cells rather than a specific and direct developmental arrest of germ cells (Russell et al. 1990).

The histology of the epididymis was normal in the homozygous mutant except that they were smaller (not shown). As expected from the testis phenotype, no spermatozoa were detected in the epididymis of $\geq 5$-week-old homozygous mutant mice (not shown).

\section{Abnormal MT morphology in ROSA63 mutant Sertoli cells}

E-MAP-115 is implicated in stabilizing and reorganizing MTs (Masson and Kreis 1993, 1995; Fabre-Jonca et al. 1999) and is expressed not only in germ cells but in Sertoli cells as well (Fabre-Jonca et al. 1998; Fig. 4D). To examine whether defects in cellular architecture could be observed in mutant Sertoli cells prior to the onset of the germ cell loss ( $\sim 5$ weeks of age), we observed the morphology of the Sertoli cell MTs at 3 weeks of age using immunofluorescence staining with an antibody against an MT subunit, $\beta$-tubulin. In heterozygous control seminiferous tubules, Sertoli cell MT bundles were detected as a spoke-like structure around the tubule (Fig. 7A). The arms of the spokes originated from around the nuclei of Sertoli cells adjacent to the basement membrane and extended toward the lumen. In the homozy- gous mutant tubule, the MT bundles were thinner and generally less well developed (Fig. 7B). Moreover, fewer numbers of detectable MT bundles were evident when observed at lower magnification (Fig. 7C,D). The numbers of Sertoli cell MT bundles per seminiferous tubule were $11.1 \pm 2.8(n=20)$ and $4.1 \pm 2.9(n=20)$ for control and homozygous mutant, respectively (Fig. 7E). Histology of the other testes of these control and mutant mice showed that at this age, the mutant testis was normal, with similar numbers of Sertoli cell nuclei observed (Fig. 7F,G). Thus, the abnormal MT morphology in the mutant is likely a primary defect. These results suggested that E-MAP-115 is required for normal morphology and function of the Sertoli cell MTs and that impaired MT functions in the Sertoli cells in the absence of E-MAP115 led to depletion of germ cells in the mutant.

\section{Discussion}

Gene-trap mutagenesis in ES cells is a powerful forward genetics approach in mice. Several groups have tried to develop methods to preselect or prescreen gene traps of interest. These genes included those induced by in vitro differentiation of ES cells (Baker et al. 1997), induced or repressed by RA (Forrester et al. 1996) and peptide growth/differentiation factors (Bonaldo et al. 1998), or encoding secretory and membrane proteins (Skarnes et al. 1995) and nuclear proteins (Tate et al. 1998). We screened for gene traps in RA-responsive genes. As the identity of RA target genes in vivo is still poorly understood, two lines of information which can be obtained from this approach (e.g., RA inducibility and loss-offunction phenotype) may help elucidate unknown targets or roles of RA in vivo. Characterization of other RA-responsive gene-trap clones is in progress.

\section{Is E-MAP-115 an RA target in the testis and other tissues?}

In animals fed a VAD diet after weaning, gradual degeneration and loss of germ cells occur in the seminiferous tubule (Mason 1933; Thompson et al. 1964). The first cell type to be lost is spermatids, which is followed by a patchy loss of spermatocytes. During this time, vacuoles associated with the Sertoli cells appear in the seminiferous tubule. Later, most of the spermatids and spermatocytes are lost and the lumen of the tubules become filled by apical extensions of Sertoli cells. At this stage, spermatogonia and a few preleptotene spermatocytes are the only germ cells detected in the tubule. The defect in VAD testis is reversed by administrating RA to VAD animals although high dosage of RA is required, suggesting that RA is the active derivative of vitamin A required for spermatogenesis (van Pelt and de Rooij 1991). Similar defects such as partial loss of germ cells and vacuolation in the seminiferous tubule are observed in $R A R \alpha$ knockout mice (Lufkin et al. 1993). No knockout mice with single or double mutations in other $R A R$ and $R X R$ genes exhibit a similar phenotype, suggesting that the effect of 
RA on spermatogenesis is mediated exclusively through $\operatorname{RAR} \alpha$.

Very little is known about RA target genes that are required for maintaining spermatogenesis. Only a few genes such as transferrin, sulfated glycoprotein-2, cytochrome c oxidase, and $R A R \alpha$ itself, have been reported to be up- or down-regulated to severalfold in the VAD testis and VAD testis replenished with retinoid (Hugly and Griswold 1987; Akmal et al. 1998; Gaemers et al. 1998). E-MAP-115 expression was up-regulated by RA both in tissue culture (ES cells) and in vivo (small intestine). In addition, it is expressed in the same cell types that express $R A R \alpha$ in the seminiferous tubule (e.g., Sertoli cells and germ cells including spermatocytes and round spermatids) (Akmal et al. 1997; Fabre-Jonca et al. 1998; Dufour and Kim 1999|. Therefore, the phenotypic similarity of ROSA63 mutant to VAD animals and $R A R \alpha$ mutant mice raised the possibility that E-MAP115 is an RA target through RAR $\alpha$ in the testis and that the defect in VAD testis is partly due to the lack of $E$ MAP-115 expression. We tested this possibility by Northern blot and in situ hybridization analyses. We failed, however, to detect significant down-regulation of E-MAP-115 expression in VAD testis and its up-regulation by retinol administration (not shown). This might indicate that E-MAP-115 expression is regulated by RA in certain tissues but not in the testis, or that RA-responsive cells are absent from the VAD testis. Consistent with this hypothesis, spermatids and most of the spermatocytes, which normally express both $R A R \alpha$ and $E$ $M A P-115$, were absent in the VAD testes in which we examined E-MAP-115 expression.

RA generally plays a key role in the differentiation of epithelia (Wolbach and Howe 1925). As E-MAP-115 is expressed in epithelial cell types of many tissues, its expression may also be regulated by RA in tissues other than testis. Indeed, its up-regulation was observed in the small intestine of VAD mice after retinol administration. In small intestine of VAD animals, morphological and functional maturation of enterocytes and goblet cells, both of which are columnar epithelial cells, is attenuated (Rojanapo et al. 1980; Warden et al. 1996). As E-MAP-115 is implicated in MT functions of differentiated epithelial cells, the defect in VAD small intestine may partly be due to down-regulation of E-MAP-115 expression. It is, however, not solely due to the down-regulation because null mutation in E-MAP-115 by itself did not affect the morphology of the tissue. This might also be true for some but not all of other E-MAP-115-expressing epithelia which are impaired in VAD animals, considering that regulation of E-MAP-115 expression by RA is tissue-specific.

Role for E-MAP-115 in MT functions in spermatogenesis

E-MAP-115 is a MAP implicated in stabilizing and reorganizing MTs. It is expressed both in Sertoli cells and germ cells in the seminiferous tubule of the testis (FabreJonca et al. 1998). Both Sertoli cells and germ cells ap- peared to be affected in the mutant, and our observations suggested a primary MT defect in each cell type. First, MTs failed to form normal manchette, leading to failure for the spermatids to elongate at the first wave of spermatogenesis. Second, Sertoli cells exhibited an abnormal MT network starting by three weeks of age, leading to eventual depletion of germ cells in subsequent waves of spermatogenesis. Why the first wave of spermatogenesis is completed is not known. It is, however, not unusual in spermatogenic mutants that the first wave of spermatogenesis occurs but is followed by massive depletion of germ cells in subsequent waves (Russell and Gardner 1974; Beamer et al. 1988).

The first defect to be observed in the mutant testis was in elongating spermatids. This phenotype suggested that the absence of E-MAP-115 is related to the failure of the manchette to develop and/or organize as the spermatid nuclei elongate and take on their characteristic configuration. Deformation of spermatid nuclei from the normal stage-related configuration is always correlated with the presence or absence of the manchette in genetic mutants such as the azh (abnormal spermatozoon head) mutant (Cole et al. 1988; Meistrich et al. 1990) and in animals treated with MT-disrupting agents such as colchicine and carbendazim (Handel 1979; Nakai et al. 1998), implicating the manchette MTs in the shaping of sperm nuclei. For example, in regions where the manchette is absent, the nuclear contour is rounded; in regions where there is ectopic manchette, the nuclear envelope is distorted and lies parallel to the manchette MTs. The manchette first appears in step eight spermatids. As the manchette abnormality and nuclear deformation of spermatids were observed at the same stages of spermiogenesis as soon as the manchette formed in the ROSA63 mutant, it is unlikely that the manchette abnormality is caused by the nuclear deformation. Thus, the ROSA63 mutant phenotype further illustrates the importance of the manchette in caudal nuclear shaping (Russell et al. 1991), and suggests an important role for E-MAP-115 in the function of manchette MTs. The possibility that the deformation of spermatid nuclei is due to a Sertoli cell defect but not to an intrinsic defect of spermatids, however, can not be completely ruled out because in one case, a defect in Sertoli cells appears to affect the morphology of elongated spermatids (Kastner et al. 1996).

The second defect, gradual germ cell loss, was likely associated with an impaired Sertoli cell function because there was no stage specificity in lost germ cells. Spermatids were first lost by three months of age, followed by the loss of spermatocytes and spermatogonia by 12 months. Consistent with this, changes in the morphology and distribution of MTs were also observed in the mutant Sertoli cells. The Sertoli cell MTs are known to play important roles in supporting spermatogenesis. First, Sertoli cells secrete the seminiferous fluid to the adluminal space of the seminiferous tubule (for review, see Griswold 1993). This fluid contains transport and carrier proteins, proteases and protease inhibitors, extracellular matrix proteins, hormones, and growth factors. These proteins are required for maintaining the nutri- 
tional and hormonal microenvironment of spermatocytes and spermatids that are inaccessible to proteins in the blood by the blood-testis barrier. MTs serve as a platform for the movement of secretory vesicles via MTmotor systems and are thus important in Sertoli cells for secreting the seminiferous fluid (Johnson et al. 1991). Second, the MTs are implicated in the movement or translocation of elongated spermatids that are tightly associated with the ectoplasmic specializations of Sertoli cells, along the axis of the MTs (Redenbach and Vogl 1991; Redenbach et al. 1992). Finally, Sertoli cell MTs are implicated in the structural integrity of the seminiferous tubule because disruption of MTs in Sertoli cells by MT-disrupting agents causes cleavage of the cells and sloughing of germ cells that are attached to the apical portion of the Sertoli cells to the lumen (Russell et al. 1981; Vogl et al. 1983). Depletion of germ cells in the ROSA63 mutant suggests that E-MAP-115 may be required in the Sertoli cells for secreting proteins that are necessary for survival and differentiation of germ cells. Vacuolation between Sertoli cells at the level of the tight junctions is an indication that the secretory system has gone astray as it suggests that the fluid secretion apparently is directed laterally, but not apically toward the lumen. We propose that MT disruption in the Sertoli cells probably leads to a constellation of events that disrupt Sertoli cell activity and result in the progressive depletion of germ cells less mature than elongating spermatids. Our results provide direct evidence for essential roles of both germ cell- and Sertoli cell-specific MTs in spermatogenesis and demonstrate an indispensable function of E-MAP-115 as a MAP for these MTs.

\section{Materials and methods}

Screening for RA-responsive gene traps

The retroviral gene trap vector ROSAßgeo* was provided by Jeffrey Hildebrand (Fred Hutchinson Cancer Research Center, Seattle, WA). The vector is similar to ROSAßgeo (Friedrich and Soriano 1991) and includes, in reverse orientation with respect to the retroviral vector $\mathrm{pGen}^{-}$, the adenovirus major late transcript splice acceptor (intron 1 /exon 2 boundary), $\beta g e o^{*}$, and the bovine growth hormone poly(A) signal, flanked by loxP sites.

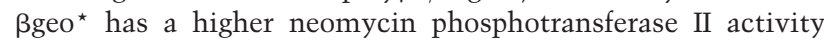
than $\beta$ geo because of a glutamic to aspartic acid conversion at residue 182 in the neo moiety (Yenofsky et al. 1990).

Retroviral infection of AK7 mouse ES cells was performed as described previously (Friedrich and Soriano 1991). Following selection with G418 for 10 days, G418-resistant colonies were picked, trypsinized, and grown on feeder cells in 96-well plates for several days. On reaching confluency, the cells were trypsinized and one sixth were plated in duplicate in gelatinized 96-well plates. One third of the cells were frozen as a master stock. When the cells in duplicate 96-well plates became $50 \%$ confluent, they were cultured in the low-serum medium (Dulbecco's modified Eagle's medium supplemented with $0.1 \%$ fetal calf serum and $2 \%$ bovine serum albumin) for $24 \mathrm{hr}$, and incubated with or without $1 \mu \mathrm{M}$ all-trans RA (Sigma) in the same medium for an additional $24 \mathrm{hr}$. Afterwards, the cells were stained with X-gal as described (Friedrich and Soriano 1991) and $\beta$-gal activity was observed under a light microscope.
Derivation, genotyping, and $X$-gal staining of mice

Blastocyst injections of ES cells were performed using standard procedures. Genotyping was performed by Southern blot analysis of DNAs from tail biopsies as described (Friedrich and Soriano 1991). DNAs were digested with EcoRI and hybridized with a neo probe. Heterozygous and homozygous mutant mice were distinguished by the intensity of the neo-positive band. A wnt1 genomic probe was used as an internal standard. X-gal staining of embryos and tissues was performed as described (Friedrich and Soriano 1991). Prior to staining, E14.5 embryos were embedded in $3 \%$ low-melting agarose in phosphate-buffered saline and cut at $400 \mu \mathrm{m}$ using a vibratome. Sex of embryos was determined by PCR for Y chromosome genes, Sry and Zfy, using yolk sac DNAs as described (Hogan et al. 1994).

\section{Cloning of cDNA}

The E-MAP-115/Bgeo* fusion cDNA was amplified from poly $(\mathrm{A})^{+}$RNA of ROSA63 ES cells by 5' RACE as described (Hildebrand and Soriano 1999). Mouse E-MAP-115 cDNAs corresponding to the amino acid residues 229-571 (nucleotide 6861712) and 574-694 (nucleotide 1720-2080) (EMBL/GenBank/ DDBJ accession no. Y15197) were amplified from wild-type ES cell poly $(\mathrm{A})^{+}$RNA by RT-PCR using the following pairs of primers: 5'-TTCTGACGCCCACACATTCG-3' and 5'-TGCTTCTCTCGTTCCTGCCG-3', and 5'-AAAGAAGAGCAGGAACGGCTGG-3' and 5'-CATTGGTGACATCTAATCTGGACG-3'.

\section{Northern blotting}

ES cells were lysed in $4 \mathrm{M}$ urea, $2 \mathrm{M} \mathrm{LiCl}$, and $1 \mathrm{~mm}$ EDTA, and incubated overnight at $4^{\circ} \mathrm{C}$. RNA was precipitated by centrifugation at $10,000 \times g$ for $30 \mathrm{~min}$ at $4^{\circ} \mathrm{C}$. The pellet was suspended in $0.1 \%$ SDS, extracted with phenol/chloroform, precipitated with ethanol, and resuspended in $\mathrm{H}_{2} \mathrm{O}$. RNA from tissues was isolated using a Trizol reagent (GIBCO BRL) according to the manufacturer's instructions. RNA $(10 \mu \mathrm{g})$ was run on a formaldehyde-denaturing gel, transferred to a nylon membrane (Hybond-N+, Amersham), and hybridized with labeled probes using standard procedures. The intensity of bands were quantified using a PhosphorImager (Molecular Dynamics).

\section{Generation of VAD mice and retinol administration}

Breeding pairs of C57BL/6J and 129/Sv mice were fed a VAD diet (Teklad Trucking) for three weeks. Male pups from these pairs received the same diet until they became VAD. At the age of 14-16 weeks when body weight decreased, mice were considered VAD. Each mouse was administered subcutaneously with $0.5 \mathrm{mg}$ of all-trans retinol (Sigma) in $50 \mu \mathrm{l}$ of ethanol, and received a normal vitamin A-containing diet (Animal Specialties) afterwards. Mice were sacrificed at 0, 24, and $48 \mathrm{hr}$ after retinol administration.

\section{Chromosome mapping}

Chromosome mapping was performed using the Jackson Laboratory BSS backcross panel with progeny from matings of $($ C57BL/6JEi $\times$ SPRET/Ei)F1 $\times$ SPRET/Ei mice (Rowe et al. 1994). Southern blot analysis of SacI-digested C57BL/6JEi and SPRET/Ei genomic DNAs with mouse E-MAP-115 cDNA (nucleotides 1720-2080) detected single 2.0-kb and $>12-\mathrm{kb}$ bands, respectively. DNAs of the progeny were therefore digested with $\mathrm{SacI}$ and hybridized with the probe to examine the 
presence or absence of these bands. The data were analyzed by the Jackson Laboratory.

\section{Histology and immunofluorescence staining}

Testes were fixed overnight with Bouin's fixative at $4^{\circ} \mathrm{C}$, gradually dehydrated in ethanol, cleared with HistoClear, and embedded in paraffin. Sections were cut at $5 \mu \mathrm{m}$ and stained by periodic acid-Shiff's (PAS) and hematoxylin staining. For immunofluorescence staining, testes were fixed overnight with $100 \%$ methanol, cleared with xylene, and sectioned as above. After removal of paraffin with xylene and rehydration, sections were stained with anti- $\beta$-tubulin antibody E7 (1:2 dilution; Developmental Studies Hybridoma Bank at University of Iowa) as described previously (Komada and Soriano 1999). The secondary antibody was a rhodamine-conjugated anti-mouse immunoglobulin G antibody (1:100; Jackson Immunoresearch). Sections were costained with DAPI. Fluorescent images were captured and processed using a Deltavision microscope (Applied Precision).

\section{Electron microscopy}

Mice were perfused with glutaraldehyde via the heart (Sparando 1990). Testes were post-fixed with osmium tetroxide and processed for plastic embedment. Thin sections were examined with a high resolution electron microscope. Staging of the spermatogenic cycle was performed according to Russell et al. (1990).

\section{Acknowledgments}

We thank Karin Weismann, Peter Mueting-Nelsen, and Philip Corrin for help with genotyping of mice; Grant MacGregor and Bob Braun for discussions and suggestions; Jeff Hildebrand for providing the ROSAßgeo* gene trap vector; Lucy Rowe and Mary Barter for assistance with chromosome mapping; and the Soriano lab members for critical reading of the manuscript. M.K. is especially grateful to Grant MacGregor for encouragement throughout. The anti- $\beta$-tubulin antibody E7, developed by Michael Klymkowsky, was obtained from the Developmental Studies Hybridoma Bank developed under the auspices of the NICHD and maintained by The University of Iowa, Department of Biological Sciences. M.K. was supported by fellowships from the Human Frontier Science Program and the Uehara Memorial Life Science Foundation. This work was supported by grant HD24875 to P.S.

The publication costs of this article were defrayed in part by payment of page charges. This article must therefore be hereby marked "advertisement" in accordance with 18 USC section 1734 solely to indicate this fact.

\section{References}

Akmal, K.M., J.M. Dufour, and K.H. Kim. 1997. Retinoic acid receptor $\alpha$ gene expression in the rat testis: Potential role during the prophase of meiosis and in the transition from round to elongating spermatids. Biol. Reprod. 56: 549-556.

Akmal, K.M., J.M. Dufour, M. Vo, S. Higginson, and K.H. Kim. 1998. Ligand-dependent regulation of retinoic acid receptor $\alpha$ in rat testis: In vivo response to depletion and repletion of vitamin A. Endocrinology 139: 1239-1248.

Baker, R.K., M.A. Haendel, B.J. Swanson, J.C. Shambaugh, B.K. Micales, and G.E. Lyons. 1997. In vitro preselection of genetrapped embryonic stem cell clones for characterizing novel developmentally regulated genes in the mouse. Dev. Biol. 185: 201-214.

Beamer, W.G., T.L. Cunliffe-Beamer, K.L. Shultz, S.H. Langley, and T.H. Roderick. 1988. Juvenile spermatogonial depletion (jsd): A genetic defect of germ cell proliferation of male mice. Biol. Reprod. 38: 899-908.

Bonaldo, P., K. Chowdhury, A. Stoykova, M. Torres, and P. Gruss. 1998. Efficient gene trap screening for novel developmental genes using IRES $\beta$ geo vector and in vitro preselection. Exp. Cell Res. 244: 125-136.

Chambon, P. 1996. A decade of molecular biology of retinoic acid receptors. FASEB J. 10: 940-954.

Cole, A., M.L. Meistrich, L.M. Cherry, and P.K. Trostle-Weige. 1988. Nuclear and manchette development in spermatids of normal and azh/azh mutant mice. Biol. Reprod. 38: 385401.

Dufour, J.M. and K.H. Kim. 1999. Cellular and subcellular localization of six retinoid receptors in rat testis during postnatal development: Identification of potential heterodimeric receptors. Biol. Reprod. 61: 1300-1308.

Elliott, D.J. and H.J. Cooke. 1997. The molecular genetics of male infertility. BioEssays 19: 801-809.

Fabre-Jonca, N., J.-M. Allaman, G. Radlgruber, P. Meda, J.Z. Kiss, L.E. French, and D. Masson. 1998. The distribution of murine $115-\mathrm{kDa}$ epithelial microtubule-associated protein (E-MAP-115) during embryogenesis and in adult organs suggests a role in epithelial polarization and differentiation. Differentiation 63: 169-180.

Fabre-Jonca, N., I. Viard, L.E. French, and D. Masson. 1999. Upregulation and redistribution of E-MAP-115 (epithelial microtubule-associated protein of $115 \mathrm{kDa}$ ) in terminally differentiating keratinocytes is coincident with the formation of intercellular contacts. J. Invest. Dermatol. 112: 216225.

Faire, K., C.M. Waterman-Storer, D. Gruber, D. Masson, E.D. Salmon, and J.C. Bulinski. 1999. E-MAP-115 (ensconsin) associates dynamically with microtubules in vivo and is not a physiological modulator of microtubule dynamics. J. Cell Sci. 112: 4243-4255.

Forrester, L.M., A. Nagy, M. Sam, A. Watt, L. Stevenson, A. Bernstein, A.L. Joyner, and W. Wurst. 1996. An induction gene trap screen in embryonic stem cells: Identification of genes that respond to retinoic acid in vitro. Proc. Natl. Acad. Sci. 93: 1677-1682.

Friedrich, G. and P. Soriano. 1991. Promoter traps in embryonic stem cells: A genetic screen to identify and mutate developmental genes in mice. Genes \& Dev. 5: 1513-1523.

Gaemers, I.C., A.M.M. van Pelt, A.P.N. Themmen, and D.G. de Rooij. 1998. Isolation and characterization of all-trans-retinoic acid-responsive genes in the rat testis. Mol. Reprod. Dev. 50: 1-6.

Gossler, A., A.L. Joyner, J. Rossant, and W.C. Skarnes. 1989. Mouse embryonic stem cells and reporter constructs to detect developmentally regulated genes. Science 244: 463-465.

Griswold, M.D. 1993. Protein secretion by Sertoli cells: General considerations. In The Sertoli cell (ed. L.D. Russell and M.D. Griswold), pp. 195-200. Cache River Press, Clearwater, FL.

Handel, M.A. 1979. Effects of colchicine on spermiogenesis in the mouse. J. Embryol. Exp. Morph. 51: 73-83.

Hildebrand, J.D. and P. Soriano. 1999. Shroom, a PDZ domaincontaining actin-binding protein, is required for neural tube morphogenesis in mice. Cell 99: 485-497.

Hirokawa, N. 1994. Microtubule organization and dynamics dependent on microtubule associated proteins. Curr. Opin. Cell Biol. 6: 74-81.

Hogan, B., R. Beddington, F. Costantini, and E. Lacy. 1994. Ma- 
nipulating the mouse embryo: A laboratory manual. Cold Spring Harbor Laboratory Press, Cold Spring Harbor, NY.

Hugly, S. and M. Griswold. 1987. Regulation of levels of specific Sertoli cell mRNAs by vitamin A. Dev. Biol. 121: 316-324.

Johnson, K.J., E.S. Hall, and K. Boekelheide. 1991. 2,5-Hexanedione exposure alters the rat Sertoli cell cytoskeleton. I. Microtubules and seminiferous tubule fluid secretion. Toxicol. Appl. Pharmacol. 111: 432-442.

Kastner, P., M. Mark, and P. Chambon. 1995. Nonsteroid nuclear receptors: What are genetic studies telling us about their role in real life? Cell 83: 859-869.

Kastner, P., M. Mark, M. Leid, A. Gansmuller, W. Chin, J.M. Grondona, D. Décimo, W. Krezel, A. Dierich, and P. Chambon. 1996. Abnormal spermatogenesis in RXR $\beta$ mutant mice. Genes \& Dev. 10: 80-92.

Komada, M. and P. Soriano. 1999. Hrs, a FYVE finger protein localized to early endosomes, is implicated in vesicular traffic and required for ventral folding morphogenesis. Genes \& Dev. 13: 1475-1485.

Lufkin, T., D. Lohnes, M. Mark, A. Dierich, P. Gorry, M.-P. Gaub, M. LeMeur, and P. Chambon. 1993. High postnatal lethality and testis degeneration in retinoic acid receptor $\alpha$ mutant mice. Proc. Natl. Acad. Sci. 90: 7225-7229.

Mandelkow, E. and E.-M. Mandelkow. 1995. Microtubules and microtubule-associated proteins. Curr. Opin. Cell Biol. 7: 72-81.

Mason, K.E. 1933. Differences in testes injury and repair after vitamin A deficiency, vitamin E deficiency and inanition. Am. J. Anat. 52: 153-239.

Masson, D. and T.E. Kreis. 1993. Identification and molecular characterization of E-MAP-115, a novel microtubule-associated protein predominantly expressed in epithelial cells. $J$. Cell Biol. 123: 357-371.

1995. Binding of E-MAP-115 to microtubules is regulated by cell cycle-dependent phosphorylation. J. Cell Biol. 131: 1015-1024.

McLachlan, R.I., C. Mallidis, K. Ma, S. Bhasin, and D.M. de Kretser. 1998. Genetic disorders and spermatogenesis. Reprod. Fertil. Dev. 10: 97-104.

Meistrich, M.L., P.K. Trostle-Weige, and L.D. Russell. 1990. Abnormal manchette development in spermatids of $a z h / a z h$ mutant mice. Am. J. Anat. 188: 74-86.

Nakai, M., K. Toshimori, K. Yoshinaga, T. Nasu, and R.A. Hess. 1998. Carbendazim induced abnormal development of the acrosome during early phases of spermatogenesis in the rat testis. Cell Tissue Res. 294: 145-152.

Redenbach, D.M. and A.W. Vogl. 1991. Microtubule polarity in Sertoli cells: A model for microtubule-based spermatid transport. Eur. J. Cell Biol. 54: 277-290.

Redenbach, D.M., K. Boekelheide, and A.W. Vogl. 1992. Binding between mammalian spermatid-ectoplasmic specialization complexes and microtubules. Eur. J. Cell Biol. 59: 433-448.

Rojanapo, W., A.J. Lamb, and J.A. Olson. 1980. The prevalence, metabolism and migration of goblet cells in rat intestine following the induction of rapid, synchronous vitamin A deficiency. J. Nutr. 110: 178-188.

Rowe, L.B., J.H. Nadeau, R. Turner, W.N. Frankel, V.A. Letts, J.T. Eppig, M.S.H. Ko, S.J. Thurston, and E.H. Birkenmeier. 1994. Maps from two interspecific backcross DNA panels available as a community genetic mapping resource. Mamm. Genome 5: 253-274.

Russell, L.D. and P.J. Gardner. 1974. Testicular ultrastructure and fertility in the restricted color (H-re) rat. Biol. Reprod. 11: 631-643.

Russell, L.D., J.P. Malone, and D.S. MacCurdy. 1981. Effect of the microtubule disrupting agents, colchicine and vinblas- tine, on seminiferous tubule structure in the rat. Tissue Cell 13: $349-367$.

Russell, L.D., R.A. Ettlin, A.P. Sinha Hikim, and E.D. Clegg. 1990. Histological and histopathological evaluation of the testis. Cache River Press, Clearwater, FL.

Russell, L.D., J.A. Russell, G.R. MacGregor, and M.L. Meistrich. 1991. Linkage of manchette microtubules to the nuclear envelope and observations of the role of the manchette in nuclear shaping during spermiogenesis in rodents. Am. I. Anat. 192: 97-120.

Skarnes, W.C., B.A. Auerbach, and A.L. Joyner. 1992. A gene trap approach in mouse embryonic stem cells: The lac $Z$ reporter is activated by splicing, reflects endogenous gene expression, and is mutagenic in mice. Genes \& Dev. 6: $903-$ 918.

Skarnes, W.C., J.E. Moss, S.M. Hurtley, and R.S.P. Beddington. 1995. Capturing genes encoding membrane and secreted proteins important for mouse development. Proc. Natl. Acad. Sci. 92: 6592-6596.

Sparando, R. 1990. Perfusion of the rat testis through the heart using heparin. In Histological and histopathological evaluation of the testis (ed. L.D. Russell, R.A. Ettlin, A.P. Sinha Hikim, and E.D. Clegg), pp. 277-280. Cache River Press, Clearwater, FL.

Tate, P., M. Lee, S. Tweedie, W.C. Skarnes, and W.A. Bickmore. 1998. Capturing novel mouse genes encoding chromosomal and other nuclear proteins. J. Cell Sci. 111: 2575-2585.

Thompson, J.N., J.M. Howell, and G.A.J. Pitt. 1964. Vitamin A and reproduction in rats. Proc. R. Soc. Lond. 159: 510-535.

van Pelt, A.M.M. and D.G. de Rooij. 1991. Retinoic acid is able to reinitiate spermatogenesis in vitamin A-deficient rats and high replicate doses support the full development of spermatogenic cells. Endocrinology 128: 697-704.

Vogl, A.W., R.W. Linck, and M. Dym. 1983. Colchicine-induced changes in the cytoskeleton of the golden-mantled ground squirrel (Spermophilus lateralis) Sertoli cells. Am. J. Anat. 168: 99-108.

von Melchner, H., J.V. DeGregori, H. Rayburn, S. Reddy, C. Friedel, and H.E. Ruley. 1992. Selective disruption of genes expressed in totipotent embryonal stem cells. Genes \& Dev. 6: 919-927.

Warden, R.A., M.J. Strazzari, P.R. Dunkley, and E.V. O'Loughlin. 1996. Vitamin A-deficient rats have only mild changes in jejunal structure and function. J. Nutr. 126: 18171826.

Wilson, J.G., C.B. Roth, and J. Warkany. 1953. An analysis of the syndrome of malformations induced by maternal vitamin $\mathrm{A}$ deficiency. Effects of restoration of vitamin A at various times during gestation. Am. J. Anat. 92: 189-217.

Wolbach, S.B. and P.R. Howe. 1925. Tissue changes following deprivation of fat-soluble A vitamin. I. Exp. Med. 42: 753777.

Yenofsky, R.L., M. Fine, and J.W. Pellow. 1990. A mutant neomycin phosphotransferase II gene reduces the resistance of transformants to antibiotic selection pressure. Proc. Nat1. Acad. Sci. 87: 3435-3439. 


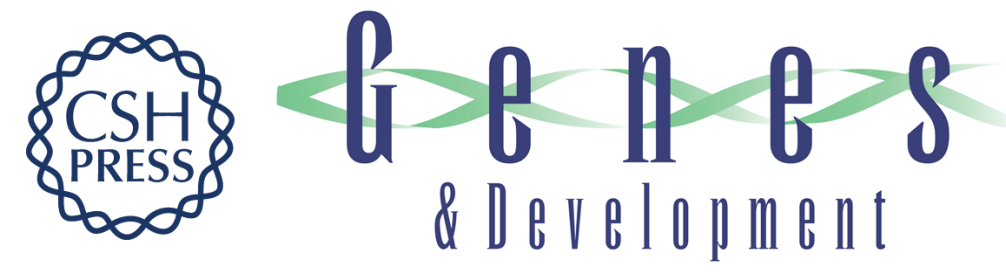

\section{E-MAP-115, encoding a microtubule-associated protein, is a retinoic acid-inducible gene required for spermatogenesis}

Masayuki Komada, Derek J. McLean, Michael D. Griswold, et al.

Genes Dev. 2000, 14:

Access the most recent version at doi:10.1101/gad.14.11.1332

References

This article cites 48 articles, 15 of which can be accessed free at:

http://genesdev.cshlp.org/content/14/11/1332.full.html\#ref-list-1

License

Email Alerting

Receive free email alerts when new articles cite this article - sign up in the box at the top

Service right corner of the article or click here.

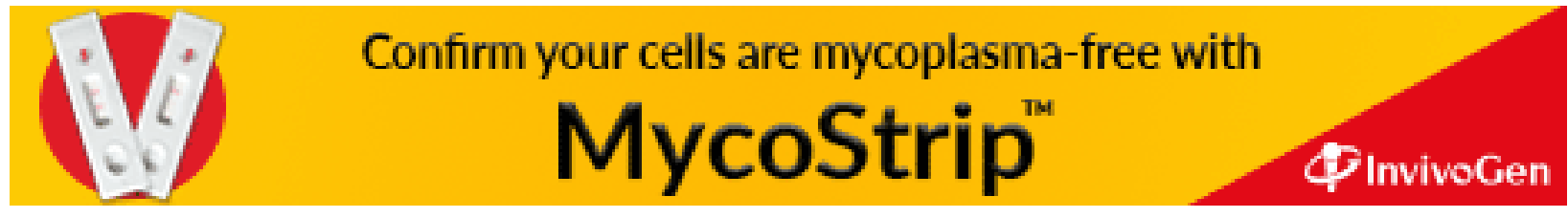

